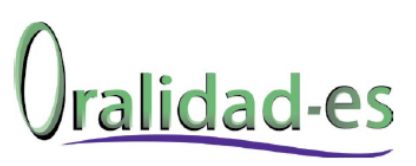

\title{
Aprendizajes discursivos pedagógicos e investigativos de maestras de educación inicial en relación con los usos orales de niños de 2 a 4 años en el marco de la pedagogía de la otredad
}

\section{Pedagogical and investigative discursive apprenticeship of initial education teachers in relation to the oral uses of children from 2 to 4 years in the framework of the pedagogy of otherness}

Angie Patricia Gómez-Garzón
angiepatog2@hotmail.com
Universidad Pedagógica Nacional, Colombia
Claudia Marcela Rincón-Wilches cmrincon@pedagogica.edu.co Universidad Pedagógica Nacional, Colombia

Mayra Alejandra Patiño-Martínez maya-le23@hotmail.com

Universidad Pedagógica Nacional, Colombia

Rincón-Vilches, C. M., Patiño-Martínez, M. A. \& Gómez-Garzón, A. P. (2017). Aprendizajes discursivos pedagógicos e investigativos de maestras de educación inicial en relación con los usos orales de niños de 2 a 4 años en el marco de la pedagogía de la otredad. Oralidad-es, 3(6), 1-17. https://revistaoralidad-es.com/index.php/ro-es/article/view/82/57 


\section{Resumen}

El propósito de este artículo "Aprendizajes pedagógicos e investigativos de maestras de educación inicial en relación con los usos orales de niños de 2 a 4 años en el marco de la pedagogía de la otredad" es socializar la investigación que desde el paradigma cualitativo, se desarrolló alrededor del campo de la primera infancia y la oralidad con niños de la Escuela Maternal de la Universidad Pedagógica Nacional (Colombia).

El ejercicio investigativo buscó a partir de las apuestas que a nivel pedagógico llevan a cabo las maestras en torno a la oralidad, donde convergen los sistemas de apoyo como andamiajes que potencian las construcciones alrededor de este campo, reconocer como los niños emplean diferentes usos orales para entablar una comunicación en los contextos dialógicos en los que se encuentran. Los hallazgos se configuran como mediadores que promueven la oralidad desde el uso que hacen los niños de esta, favoreciendo el reconocimiento del otro desde sus diferentes formas de comunicarse a partir de una perspectiva sociocultural.

\section{Palabras clave}

Oralidad; usos orales; primera infancia; proceso de alteridad; didáctica; investigación cualitativa

\section{Abstract}

The purpose of this article "Pedagogical and investigative learning of early education teachers in relation to the oral uses of children from 2 to 4 years in the framework of otherness pedagogy" is to socialize the research that from the qualitative paradigm, was developed around the field of early childhood and orality with children from the Maternal School of the National Pedagogical University (Colombia).

The investigative exercise sought from the bets that at pedagogical level carry out the teachers around the orality, where the systems of support converge as scaffolds that promote the constructions around this field, to recognize as the children use different oral uses to establish a communication in the dialogical contexts in which they are. The findings are configured as mediators that promote orality from the use that children make of it, favoring the recognition of the other from their different ways of communicating from a socio-cultural perspective.

Key words

Orality; oral uses; early childhood; otherness process; didactics; qualitative research 


\section{Introducción}

En este artículo, se presentan los resultados de la investigación "Sistemas de apoyo que potencian la oralidad de los niños de la Escuela Maternal de Universidad Pedagógica Nacional" avalada por el Centro de Investigaciones de la Universidad Pedagógica Nacional (CIUP, Colombia) durante el 2017. Es llevada a cabo en la Escuela Maternal de la Universidad Pedagógica Nacional (UPN) ${ }^{1}$ , escenario de educación no formal con niños de 2 a 4 años por tres maestras egresadas de la Licenciatura de Educación Infantil de la UPN con el acompañamiento de una profesora de la Facultad de Educación de la universidad ${ }^{2}$. Las maestras en mención hacen parte del grupo de investigación categorizado en Colciencias "Educación Infantil, pedagogía y contextos" del Proyecto Curricular de Educación Infantil de la Universidad Pedagógica Nacional.

La problemática que abre paso a la investigación, según el estudio de antecedentes, identifica cómo el campo de la oralidad con niños menores de cuatro años ha sido poco abordado en la investigación a nivel pedagógico. Por consiguiente, el rol del maestro con esta población infantil se reduce a los cuidados asistenciales.

A partir de este reconocimiento, se infiere que no habría razones para abordar de forma sistemática la enseñanza de la oralidad en los contextos de socialización diferentes a la familia, se desconoce, por una parte, el papel del maestro como mediador en la estructuración de la lengua materna y sus necesarios andamiajes para el aprendizaje de la oralidad en los primeros años y por otra, la importancia de la escucha, el componente paralingüístico y el contexto en la estructuración de la significación (timbre de voz, las pausas para cambiar de turno, remarcar entonaciones, el tono que da cuenta de estados de ánimo, el volumen que transmite emociones, el ritmo, el carácter de la situación comunicativa).

Además, los puntos de referencia para que los maestros aborden la oralidad en el aula se retoman desde la medicina o la terapia del lenguaje, campos de estudio con gran cantidad de elaboraciones centradas en miradas desde el déficit del habla, con imaginarios de infancia que asumen al niño como un sujeto carente, indefenso, ignorante e incapaz que no tiene "qué decir" porque aún "no habla de forma estructurada" ${ }^{4} \mathrm{y}$ la inexistencia del maestro como profesional de la educación.

En relación con el corpus a nivel pedagógico, son mínimas las elaboraciones en Colombia sobre oralidad en la primera infancia ${ }^{5}$. Básicamente ha sido desarrollado por Yolanda Reyes $(2010)^{6}$ para el contexto educativo no formal. En cuanto al contexto educativo formal, hay investigacio-

\footnotetext{
${ }^{1}$ Escuela Maternal: Institución de innovación cuyo propósito es apoyar en el cuidado y la formación de hijos de funcionarios, estudiantes, contratistas y docentes vinculados a la Universidad Pedagógica Nacional, Colombia que se encuentran en edades entre los 4 meses y los 4 años.

${ }^{2}$ Claudia Marcela Rincón.

${ }^{3}$ Concepto tomado de Bruner referido al proceso de enseñanza que facilita el aprendizaje a través de pequeños pasos comprensibles hacia la consecución de una meta, apoyados progresivamente por un adulto o un par más capaz

${ }^{4}$ Según las investigaciones desarrolladas en la Licenciatura de Educación Infantil de la UPN en el 2013: Investigación "Prácticas pedagógicas de las egresadas de la Licenciatura de Educación infantil de la Universidad Pedagógica Nacional Colombia que promueven la oralidad en niños y niñas menores de cinco años" avalada por la Facultad de Educación de la UPN durante 2012-2013 y elaborada por los profesores del grupo de Comunicación, lenguaje e Infancias y en el 2015 el estudio documental sobre la oralidad en la primera infancia en Bogotá. Autoras: Universidad Pedagógica Nacional (Colombia) noviembre 2015.

En el Proyecto Curricular de Educación Infantil de la Universidad Pedagógica Nacional, se asume la primera infancia como la población menor de 6 años que hace parte tanto de la educación formal como de la no formal.

Desde la Secretaría Distrital de Integración Social a través del Lineamiento Pedagógico y Curricular para la Educación Inicial en el Distrito (2010), la autora plantea tres momentos para la adquisición del lenguaje en la primera infancia, estos son: la conquista del lenguaje humano, el acercamiento progresivo al lenguaje verbal y posteriormente al lenguaje escrito. De forma puntual la autora presenta avances en torno a elementos verbales y no verbales que enmarcan las primeras "conversaciones" del bebé, como la primera aproximación a las palabras, que finalmente le permita llegar a la estructura convencional del lenguaje.
} 
nes y experiencias pedagógicas rigurosas ${ }^{7}$, pero centradas en niños mayores de cuatro años y son pocas con respecto al amplio abordaje que tiene la investigación pedagógica sobre lectura y escritura, evidenciando la jerarquización de estas dos actividades discursivas con respecto a la oralidad.

De este modo, las prácticas pedagógicas de las maestras para la enseñanza y el aprendizaje de la lengua materna, específicamente de la oralidad, se caracterizan ${ }^{8}$ por ser prácticas que promueven la oralidad pero de manera no intencionada, sin mayores claridades conceptuales, sin ningún desarrollo sistemático en planeaciones e intervenciones pedagógicas, muchas veces son simples y aisladas, pensadas desde una perspectiva instrumental de la didáctica ${ }^{9}$ y por consiguiente, en palabras de Rodríguez (2002), no se constituyen en un Sistema de Apoyo Pedagógico. Prácticas que no van más allá de usar el sistema de apoyo natural -LASS- (Bruner, 19) que ofrece la madre $\mathrm{u}$ otro adulto de manera intuitiva.

A esta problemática se adiciona la escasa apertura en las instituciones educativas, de espacios de encuentro pedagógico y socialización ${ }^{10}$ en los que las maestras puedan abordar sus preguntas, la toma de decisión y la estructuración de la práctica pedagógica en relación con la enseñanza y el aprendizaje de la oralidad con niños menores de cuatro años.

De forma puntual, el objetivo general de la investigación es reconocer los usos orales que se configuran en la Escuela Maternal de la Univer- sidad Pedagógica Nacional con los niños de 2 a 4 años a partir de los sistemas de apoyo didáctico estructurados por las maestras. De esta forma, la investigación contribuye a hacer evidente la importancia de la oralidad en la primera infancia ya que es por medio de esta que inician la construcción de la referencia, organizan y recrean la realidad y se configuran como sujetos sociales, culturales, gracias a los procesos de intersubjetividad, procesos en los que se hará especial énfasis en este escrito. Igualmente visibilizar el papel del maestro en el desarrollo de la misma y la necesidad de aportar al campo del lenguaje a nivel pedagógico, así como las articulaciones entre la Licenciatura de Educación Infantil de la UPN y la Escuela Maternal a partir del acompañamiento a sus egresados, intencionalidad emergente en las tendencias educativas mundiales.

\section{Desarrollo teórico o conceptual de la investigación}

Las apuestas teóricas para este proyecto de investigación se construyen en relación con el campo del lenguaje, la oralidad y los sistemas de apoyo como mediaciones desde la perspectiva discursiva, cognitiva y sociocultural configurada a partir de los desarrollos teóricos de Vigostky (2000) y Bruner (2001). También se retoma a Halliday (2013), Van Dijk (1978), Gartón (1994),

\footnotetext{
${ }^{7}$ Investigaciones del grupo de Identidad y Cultura de la Universidad Distrital de Colombia.

Las investigaciones desarrolladas por la Universidad del Valle, Colombia.

Las experiencias pedagógicas y de investigación en el aula de maestros que conforman el Nodo Centro, Valle y Tolima de la red de lenguaje, Colombia. Las experiencias de investigación de la red iberoamericana de oralidad.

${ }^{8}$ Según los resultados de investigación del grupo de profesores de la Licenciatura de Educación Infantil de la UPN "Prácticas pedagógicas de las egresadas de la Licenciatura de Educación infantil de la Universidad Pedagógica Nacional (Colombia) que promueven la oralidad en niños y niñas menores de cinco años” avalada por la Facultad de Educación de la UPN durante 2012-2013 y elaborada por los profesores.

${ }^{9}$ Asumida en este proceso investigativo como campo de acción, reflexión y recontextualización de saberes pedagógicos y disciplinares. En este sentido, la mirada está dirigida no solo al qué y al cómo sino hacia el por qué y el para qué, concediéndole una especial importancia "al saber" como una construcción social que se da intencionalmente en un contexto cultural particular. La pregunta por el cómo y el qué entonces, se resignifica en una constante reflexión sobre la práctica.

${ }^{10}$ Se refiere a un día al mes para abordar todas las problemáticas que van surgiendo en la institución, denominada "jornada pedagógica" lo cual no posibilita un proceso sistemático de seguimiento para trabajar en torno a las practicas pedagógicas y cómo promover la oralidad.
} 
Baena (1996), Bajtin (1998), Cestero (1998), Tusón (2001), Calsamiglia y Tusón (1999) (2002), Núñez (2003), Camps (2004), (2005), Lomas (2006) y las investigaciones del colectivo de profesores de Comunicación, lenguaje e Infancias ${ }^{11}$ de la Licenciatura de Educación Infantil de la UPN(Colombia), (2001), (2004), (2008), (2013) (2 $^{12}$ y del grupo Lenguaje, Identidad y Cultura de la Universidad Distrital Francisco José de Caldas (Colombia) (2002), (2008), (2009).

Se eligieron estos autores porque han estudiado el lenguaje desde una dimensión social y cultural y de esta forma reconocen desarrollos específicos orales de acuerdo a los contextos particulares en donde se desenvuelven los niños y los adultos; de igual manera, la mayoría de los autores mencionados han destacado dentro de sus investigaciones el papel de la escuela y más específicamente del adulto en la adquisición y desarrollo de la oralidad.

Con respecto a otras categorías como la intersubjetividad, la conversación, el juego y la perspectiva didáctica, se abordaron autores como Rogoff (1993), Garton (1994), Jolibert (1999), Litwin (1997), Camps (2004), Silvestri y Blanck (1993), Calsamiglia y Tusón, (1999) que las desarrollan en el marco de la perspectiva antropológica, discursiva, cognitiva y sociocultural. En cuanto a la categoría de infancias, los desarrollos se hacen desde las elaboraciones de Postman (1982), Narodowski (1999) y Amador (2012).

El componente de la investigación que tiene que ver con el maestro y sus creencias, los procesos de transformación de sus prácticas pedagógicas, se trabaja teóricamente desde la reflexión, como dispositivo que potencia la permanente actualización y transformación de los maestros, retomando para ello los aportes de Schon (1997), Giroux (2003), Tardif, M (2004), Perrenoud, P (2006), Marcelo (1987) y Camps (2004).

\section{Metodología empleada}

En relación con el componente metodológico, la investigación se enmarca en la investigación cualitativa. De manera específica se retoma la investigación colaborativa como una modalidad de la investigación-acción. Se busca caracterizar e interpretar una realidad y con base en los análisis resultantes, favorecer la reflexión conjunta de las maestras de la Escuela Maternal que resignifique sus apuestas pedagógicas en torno a los sistemas de apoyo didáctico que potencian la oralidad y los usos orales de los niños, a partir del análisis de tres entrevistas semiestructuradas, nueve registros de video de las sesiones pedagógicas con los niños y los conversatorios entre las maestras, que se interrelacionan con los tres momentos de la ruta metodológica los cuales son: Leyéndome a mí misma y leyendo a otras, Construyendo juntas y Construyendo autónomamente ${ }^{13}$.

\section{Resultados de la producción de conocimiento}

Los hallazgos se organizaron a partir de tres componentes: los usos orales empleados por los niños en el marco de intencionalidades comunicativas, los sistemas de apoyo que usan las maes-

\footnotetext{
${ }^{11}$ Este equipo hace parte de la línea en formación, pedagogía y didáctica del grupo de investigación Educación Infantil, Pedagogía y Contextos el cual se encuentra inscrito en Colciencias (Colombia).

12* Formas de ayuda que emplea el educador infantil para favorecer el desarrollo del lenguaje en su función cognitiva en niños de 3 meses a 5 años (2001).

* Construcción de una propuesta didáctica para favorecer el desarrollo del lenguaje en su función cognitiva: una propuesta de formación docente sustentada en la investigación (2004).

* Construcción de sistemas de apoyo didáctico para favorecer el desarrollo del lenguaje con niños menores de tres años (2008).

${ }^{13}$ Estrategias producto de la investigación: Construcción de una propuesta didáctica para favorecer el desarrollo del lenguaje en su función cognitiva: una propuesta de formación docente sustentada en la investigación (2004). Realizada por las profesoras de la Licenciatura de Educación Infantil.
} 
tras y el proceso reflexivo, metacognitivo de las maestras que genera la investigación, gracias al cual, se promueve la confrontación y transformación de los saberes.

En relación con el primer componente, a través de los tres momentos propuestos metodológicamente, se identificaron los siguientes usos orales de los niños de caminadores II y Aventureros I, como parte del trabajo pedagógico intencionado de las maestras para potenciar la oralidad: $L a$ repetición literal como una manera de interiorizar elaboraciones orales de la maestra con el fin de exponerlas en distintas situaciones comunicativas con sus pares como parte de la construcción de su oralidad; la pregunta, que utilizan para interpelar y potenciar la intersubjetividad; $l a$ onomatopeya, de la cual hacen uso para reafirmar la referencia, y los acercamientos a la narración que algunos de los niños se han retado a utilizar como una forma de exponer una situación de interés y explicar algún acontecimiento.

Respecto a la categoría de intersubjetividad, la construcción de la otredad por parte de los niños de la Escuela Maternal de la UPN se evidencia de forma preponderante en situaciones de conflicto, en donde existen golpes o empujones por el deseo de obtener algo o por la necesidad de comunicar molestia o incomodidad a sus pares por alguna de sus acciones, de esta manera, la maestra es un agente fundamental en esta adquisición paulatina de los niños, pues es quien a través del préstamo de conciencia, los sitúa en situaciones de interacción que les permiten comprender el lugar del otro y en esa medida, crear conciencia de la importancia de establecer límites en su relación con los demás y acercarse a prácticas de cuidado.

Así, fue posible evidenciar en los videos que fueron insumos de esta investigación, cómo para los niños menores de 2 años, los golpes y empujones son acciones que utilizan para demostrar molestia y conseguir diferentes elementos de su interés, pues la palabra aun no es el medio aser- tivo que les permita mediar y establecer límites para sí y para quienes le acompañan, razón por la cual, la maestra se vuelve fundamental en la mediación de procesos de conciencia en torno al reconocimiento de los demás:

El préstamo de voz de la maestra es utilizado en una situación de conflicto en donde una niña muerde a otra:

M3: “¿Por qué la mordiste Avril? Ya hablamos que para eso no son los dientes" negando con su dedo "ayayai mira, la lastimaste" consintiendo a la otra niña (...) "ipara qué se usan los dientes?"

N2: acerca sus manos a su boca, denotando la acción de comer.

M3: rescata el gesto del niño "para comer" haciendo sonidos con su boca " $\mathrm{mmmm}$, para comer, no para lastimar a los amigos" mirando a Avril.

N1: la niña sigue llorando.

M3: "ya, cálmate, yo sé que te dolió, hay que decirle a la amiga que no" negando con su dedo y mirando a Avril.

En esta situación, la maestra genera un espacio en donde los niños llegan a ser partícipes y protagonistas de la construcción de la intersubjetividad, pues al exponer lo sucedido ante todo el grupo, los moviliza a pensar en el lugar del otro como un sujeto que siente, por lo que en el ejemplo citado, la maestra retoma tanto el lugar de quien dio el golpe, como de quien lo recibió, llevando al primero a la reflexión frente al uso de los dientes y a su manera de emplearlos en otros momentos, haciéndolo consciente de la consecuencia que generó su acción. En cuanto al segundo, la maestra le muestra a la niña cómo a través de la palabra puede establecer un límite desde donde se valide su lugar y su deseo de no ser agredida, dejando por sentado, la formas a 
través de las cuales es posible asumir una relación entre pares y con ello el reconocimiento de un colectivo que le acompaña.

Es así como estos espacios de conversación frente a la construcción de la otredad por parte de los niños, paulatinamente, les da la posibilidad de asumirse como sujetos activos en ese proceso, portadores de una voz propia, a partir de la cual pueden posicionarse frente al otro:

M3: pasito, pasito, mira Amelia dile no.

$N: n o$.

M3: no, ay yayay.

N: no, ay yayay.

M3: me lastimas!

$N$ : me lastimas, ayayay!

M3: escucha lo que te está diciendo Amelia Thomas.

$N$ : ayayay.

M3: a ver, Thomas va escuchar lo que le va a decir Amelia.

$N$ : ayayay.

M3: ven que Amelia quiere decirte una cosa Thomas. Amelia habla con Thomas (...) Amelia te está diciendo que la estás lastimando, que eso no se hace.

N: lastimando.

Para este ejemplo, es claro cómo el andamiaje que hace la maestra a través de su voz, moviliza a la niña a quien lastiman, a expresar su molestia por medio de su oralidad, además de establecer un límite claro frente al cuidado que asume de sí misma. De igual manera, logra convocar a quien la lastima con el fin de que este niño logre escucharla y hacerlo consciente de su acción, siendo la maestra quien promueve un espacio adecuado en el que pueden expresar su sentir frente a lo sucedido y, en esa medida, reconocerse.

Finalmente, se logra evidenciar cómo los niños en la medida en que apropian estos medios de emplear su oralidad en situaciones de conflicto, les permiten llegar a mediar de forma cada vez más autónoma estas acciones y así participar activamente en la construcción de intersubjetividad:

M2: "Alejandro, ¿tú por qué le estas pegando a Thiago?"

N1: "Thiago".

M2: "No, esa no es la forma de tratar a tu amigo Alejo".

N2: mira a Alejo y le dice: "Alejo no" negando con su dedo.

M2: "Mira, escucha a Valery que te está diciendo: Que esa no es la manera Alejandro".

En este ejemplo, es claro cómo una de las niñas del grupo se sintió motivada a participar desde su oralidad en la construcción de conciencia sobre el cuidado por el otro, haciendo explícito desde su participación que no se debía pegar, por lo que la maestra valida estas construcciones y las amplía aclarando que los golpes no son la manera de tratar a los demás. De esta forma, la participación de la niña frente a la situación de conflicto permitió que fuera ella quien prestara conciencia a sus pares a través de su voz en torno a la importancia de cuidar a los otros desde el reconocimiento que progresivamente logren de los demás, asumiéndose como un sujeto activo en estos procesos y jalonando la construcción de la intersubjetividad en los distintos contextos de interacción en los que participe.

Se puede concluir que los procesos de alteridad se van consolidando en la medida en que la oralidad y los puentes que el maestro realiza alrededor de la misma le permiten al niño validar al otro como un agente fundamental en su cons- 
trucción como sujeto. Allí la palabra empieza a aparecer intencionalmente con el propósito de que ese otro logre conocer las molestias, deseos o necesidades de quienes las expresan, posicionándose como un locutor-interlocutor válido en los procesos comunicativos que se dan.

Con respecto al segundo componente, los sistemas de apoyo que usan las maestras para potenciar la oralidad de los niños se han identificado como hallazgos en el préstamo de conciencia a través de la voz de la maestra y de los pares, gracias a la cual fue posible asumir la repetición literal y la recontextualización en otras situaciones, como parte de la ampliación de las construcciones de los niños, haciendo paráfrasis de sus elaboraciones y complejizándolas durante la interacción. Es una acción pedagógica construida a partir de una relación de alteridad, una relación ética basada en el reconocimiento del otro desde sus diferentes forma de manifestarse y comunicarse, todo ello en el marco de una pedagogía del Nos-Otros (Ortega, 2012).

Como parte de dicho préstamo de conciencia, aparece la pregunta que se estructura a partir de los niveles de desarrollo de los niños, pues su intención varía por parte de la maestra para favorecer la oralidad, así, por ejemplo, fue evidente la presencia constante de la pregunta cerrada en el grupo de caminadores II, pues allí, su intención es ser parte activa de la construcción de referencia en los niños y para el caso de Aventureros I, este tipo de pregunta, se utiliza como una forma de validar y afirmar las elaboraciones de los niños frente a un referente para dar paso a preguntas de tipo abierto que los lleven a otras construcciones más complejas en la ampliación de dicho referente. En este sentido, se ubica como segundo hallazgo, la conversación como protogénero que favorece otros géneros discursivos y por lo tanto, la oralidad formal, siendo un espacio potente en torno a la escucha, la construcción de la otredad, el principio de alteridad que permite entablar procesos dialógicos con la primera infancia y determinar las diferentes formas de interacción entre los niños y sus maestras según la apropiación que van realizando de la oralidad. Gracias a ella los niños logran participar desde sus construcciones orales propias y los sistemas de significación que acompañan la palabra. De esa manera, la conversación se convierte en un espacio de interés para los niños, en donde pueden evidenciar ideas y emociones que suscitan alguna situación cotidiana. Allí, es importante el reconocimiento de los otros participantes de la conversación desde la conciencia paulatina del turno como una forma da validar la intervención de los demás y reconocerla como parte de la propia.

En relación con este componente, el juego se evidencia como un tercer hallazgo frente a los sistemas de apoyo, siendo el puente entre lo pedagógico y lo lingüístico. Funciona como andamiaje de la oralidad, pues se convierte en un espacio libre en donde los niños logran actuar de forma espontánea alrededor de los juegos que propician, siendo necesario para ellos expresar frente a la acción con los objetos o con sus pares. Por lo tanto, la maestra es una mediadora en los juegos de los niños, no invadiéndolos, sino acompañando sus intereses desde el préstamo de conciencia a través de su oralidad. Esto les posibilita hacer búsquedas en su oralidad que a su vez, les permite construir elaboraciones en relación a aquello que están disfrutando, que les genera interés y los motiva a intervenir desde sus procesos orales particulares.

Agregado a ello, se ubica la narración como un cuarto hallazgo, pues hace parte importante de la interacción de la maestra con los niños, al promover su interés y necesidad por expresar frente a alguna situación, encontrando en ello la oportunidad para acercarse a procesos de secuencia lógica de los hechos y búsquedas en procesos de explicación que les permitan participar de forma más activa. 
En este sentido, para el caso de Aventureros $\mathrm{I}^{14}$, la construcción de significado por parte de los niños se modificó en relación al uso de su oralidad en situaciones cotidianas de forma mucho más autónoma y como parte fundamental para comprender y asumir situaciones de conflicto, motivándolos a participar activamente de procesos de cuidado en el reconocimiento de la otredad en el que se encuentran.

Las maestras de forma intencional buscan la construcción de sentido a partir de un trabajo sistemático sobre la palabra acompañada del gesto, de la representación activa e icónica teniendo en cuenta que con niños menores de tres años la interacción se centra en la comunicación bidireccional entre el maestro y el niño. Por consiguiente es mayor el préstamo de conciencia a través de su voz que el docente debe realizar en la medida en que muchos de los niños y niñas con los que interactúan aún no hablan de forma completa y para construir la significación se apoyan en estos sistemas de representación. Allí se observa la diferencia de la comunicación que se establece con niños mayores de tres años, en la cual prima la interacción entre los niños por encima de la interacción que estos establece con la maestra, es menos dependiente de esta; por lo tanto, el préstamo de conciencia a través de su oralidad disminuye.

Finalmente, el tercer componente tiene que ver con el proceso reflexivo, metacognitivo, de las maestras, gracias al cual, se fue resignificando lo que se dice y se hace. Al hacer el análisis de este componente se identifica que el proceso da cuenta de logros significativos gracias a varios factores. Por un lado, al sentido de cada uno de los momentos de la ruta metodológica y los retos que promueve por otro, gracias a las mediaciones que se abordaron para favorecer la reflexión. Es decir, los conversatorios, el análisis individual y colectivo de los registros de video, las lecturas de materiales que daban claridades conceptuales a las preguntas emergentes de la práctica diaria con la intención de promover la oralidad y a través de ella la intersubjetividad.

Con respecto al primer factor:

Profesora 1

[...] Fue importante para esta tercera etapa volver sobre los aprendizajes que se construyeron en el primer y segundo momento, como una manera de consolidar en una propuesta autónoma, los saberes, los intereses, las intenciones que acompañan al maestro en el trabajo pedagógico con los niños, las niñas y las familias [...] diseñar una propuesta de intervención con las familias; aspecto que me reto a tener claridades conceptualmente respecto a lo que quería compartir, asi como a darme la posibilidad de reconocer las historias que acompañan a los papás y mamás del grupo y a entender las prácticas orales que entablan con sus hijos, en esa medida aportar a procesos de consciencia, donde se enriqueciera la oralidad de los niños y las niñas...”

\section{Profesora 2}

"[...]los tres momentos de la investigación (Leyéndome a mí misma y leyendo a otras, Construyendo juntas y Construyendo autónomamente)bajos los cuales retroalimentamos las intervenciones con los niños desde los espacios de conversación entre quienes participamos, fueron bastante pertinentes, en tanto, posibilitaron evidenciar claridades frente al trabajo pedagógico de la oralidad con niños menores de dos años, desde la realización de lecturas y análisis de videos de las prácticas de las maestras, lo que permitió enriquecer las apuestas de cada momento desde la retroalimentación de los pares, contribuyendo asi a propuestas futuras[...]"

\footnotetext{
${ }^{14}$ Niños de dos a dos años y medio.
} 
Profesora 3

"[...]los momentos del proceso, fueron decisivos para lograr hacer un cambio real en mi ser maestra. El primero de ellos, aunque confrontante, ya que mostrar a otros lo que se realiza en el aula y exponerse ante los mismo no es algo fácil, permitió reconocer aquellas estrategias $y$ andamiajes que se empleaban en la relación comunicativa con los niños $y$ las niñas, tomando mayor consciencia alrededor de aquellas acciones que se llevaban a cabo con los mismos.

"[...]En el segundo momento no solo se seguiría enriqueciendo las experiencias llevadas a cabo en el aula, sino que se pondrían en discusión con las diferentes maestras que hacen parte de la investigación, quienes con su experiencia cualificarian lo que se propone realizar (...) empezaria ahondar y entender cada vez más lo que era el préstamo de conciencia a través de la voz de la maestra..."

“[...]En el tercer y último momento, tendríamos que ponen en juego las construcciones que se han hecho a lo largo del proceso, punto en el cual, cada una de las secuencias didácticas propuestas debian reflejar aquellos aprendizajes, develar las cambios que se han dado..."

En relación con el segundo factor:

Profesora 1

"[...]Uno de los elementos que considero nutrió enormemente mi quehacer docente fue la oportunidad de reflexionar en torno a la secuencia didáctica pues fue muy importante para mi detenerme en cada momento $y$ pensar que quería potenciar desde el lenguaje $y$ como lo iba hacer, esto me permitió tener claridades sobre lo que iba a desarrollar en términos pedagógicos..."
"[...] ha sido muy interesante reconocer mediante los videos de cada maestra, aquellas características propias que hacen que la practica educativa sea tan diversa y potente en todas, llegar a identificar esos elementos constituye no solo para mí sino para mis compañeras un reconocimiento de ese ser maestra que se ha venido construyendo, pensando y transformando"

\section{Profesora 2}

"[...] considero que la oralidad ha sido la vía principal por la cual he transitado este camino, pues los conversatorios desde los cuales se ponen en evidencia las inquietudes, comentarios, sugerencias y demás, han propiciado diálogos entre quienes participamos, los cuales han enriquecido enormemente nuestro accionar con los niños además de continuar formándonos en torno a la reflexión como un dispositivo esencial en la cualificación de la práctica docente. Por tanto, participar de un espacio en el cual la voz de los participantes es válida y prioritaria en las construcciones e intercambio de saberes, ha sido una nueva forma de resignificar la oralidad propia, situándola como parte de la expresión de ideas y emociones que permean al docente, cuya cualificación es posible también desde la mirada del otro que busca contribuir desde sus particularidades de su ser y hacer con los niños..."

"[...]fue posible consolidar conciencia frente a la importancia del trabajo pedagógico de la oralidad con la primera infancia (...) Esta claridad, permitió enriquecer cada vez más las planeaciones que como maestras propusimos, pues conllevó a la necesidad de hacer conciencia de los momentos bajo los cuales intervenir, las estrategias a utilizar, la disposición de espacios, la escogencia de ma- 
terial, entre otros, teniendo como finalidad, contribuir a la potenciación de la oralidad de los niños y niñas de forma cada vez más asertiva..."

Así, conversar sobre los hallazgos emergentes gracias a las experiencias promovidas en la práctica, posibilita que las maestras reconozcan lo que potencian sus prácticas pedagógicas, lo que les lleva a encontrar en la lectura de sí mismas, de los otros y con los otros, nuevas formas de mirar lo pedagógico y trabajar en la cualificación de sus prácticas a través de la investigación, resignificando el sentido que esta última tiene como posibilidad de aprendizaje continuo "reflexionar sobre la propia práctica también significa reflexionar sobre la propia historia, los habitus, la familia, la cultura, los gustos, y aversiones, las relaciones con los demás, las angustias y las obsesiones" (Perrenoud, 2006, p.58).

De allí que la reflexión se posicione como un procesos de conciencia, que en el maestro permiten cualificar su formación, ampliar su saber en torno a aquellos campos vitales en los que se mueve el niño, un saber pertinente para el trabajo con la primera infancia, aquella etapa de la vida que se ha dejado relegada, contribuyendo al cambio de mirada sobre los niños menores de tres años, donde la maestra propone un trabajo intencionado y pertinente, Camps (2004, p. 10) en este sentido expone que:

Las actividades se llevan a cabo a través de acciones a las cuales los agentes implicados confieren finalidades conscientes; es decir las acciones implican intencionalidad $y$, por lo tanto, anticipación del resultado, del efecto esperado, en el marco de la interacción social en que se desarrolla la actividad.
En este sentido, se puede reconocer cómo el proceso reflexivo le dio la posibilidad a las maestras de hacer transformaciones a su accionar pedagógico y a su ser docente, cambios que permiten asumir una postura diferente en la cotidianidad del escenario educativo, observando la trascendencia de este tipo de escenarios, en el que el maestro se encuentra para dialogar sobre lo que le acontece, con miras a impactar su práctica en el aula de manera permanente.

Algunos aprendizajes puntuales obtenidos a través de la investigación:

\section{Profesora 1}

"[...]gracias al abordaje teórico y a las constantes reflexiones que realizamos con el grupo, surgió un interés como maestra frente a la escucha; elemento importante que hace parte del proceso de oralidad del ser humano, de alli comprendí que para potenciar y enriquecer el lenguaje oral en los niños se hacía indispensable aprender a escucharlos, validar su voz y darles un lugar en el espacio que compartíamos con los otros, es asi que no sólo como maestra debía darme a la tarea de ser más receptiva, también debia hacer consciencia a los niños de que su voz era importante, de motivarlos a participar $y$ dar sus aportes, en esa medida requería de unos interlocutores que escucharán lo que sus compañeros tenian por decir, porque para poder hablar se necesita de ese otro que me escuche, que me refute, que me apoye, que construya conmigo el saber pero ante todo que me reconozca como un sujeto social..."

\section{Profesora 2}

"[...] una experiencia trasformadora como maestra, pues me ha motivado a cuestionarme constantemente por mis prácticas $y$ situarlas en un marco de reflexión, en donde 
la palabra del otro se convierte en fundamental para la cualificación de mi proceso. Por ello, participar de un espacio como éste, ha sido un reto, pues ha implicado ampliar saberes teóricos que den piso a la experiencia con los niños, y de esta forma, contribuir a los procesos orales del grupo de Aventureros I de forma mucho más asertiva..."

\section{Profesora 3}

[...] debemos empezar por resaltar que la mirada alrededor de la oralidad ha cambiado, ya que se han ampliado aquellos conocimientos incipientes que se tenían al inicio del proceso. De all, que ya no se asuma solo como el acto de hablar por sí solo, sino por el contrario, se reconoce como un sistema integrado, en el cual convergen canales como el paralingüístico, el no lingüístico y lo proxémico.

"[...] enfrentar los miedos y la incertidumbre que generaba trabajar con la infancia de un año de edad, aquellos niños que al no haber conquistado en su totalidad la palabra hablada, tenían otras formas de comunicación, que hasta no ser abordadas a profundidad en el proceso de mentorazgo no fueron tan notorias para mí. En este sentido, el diálogo que se empezó a entablar tuvo otro sentido, $y$ ahora partía desde lo paralingüístico y lo gestual, lenguajes que se fueron enriqueciendo y potenciando para darle sentido a aquellas construcciones que venían haciendo los niños y las niñas. De alli que la lectura de un cuento, la exploración de nuevos elementos, la realización de una receta, entre otras acciones pedagógicas, fueran dispuestas de forma diferente, donde se acompañaban de lo icónico, simbólico y enactivo para dar un nuevo significado a lo realizado, gestando intencionalmente situaciones para propiciar una comunicación...”

\section{Conclusiones}

A través de este apartado se quiere reconocer que han sido muchos los aprendizajes. Unos en relación con los usos orales más empleados por los niños y como desde esta estructuración inicial de la oralidad se aporta pedagógicamente a la configuración no solo de la intersubjetividad sino de forma holística a la construcción referencial y estética del mundo. Otros aprendizajes corresponden al campo pedagógico en relación con la capacidad de las maestras para continuar aprendiendo y asumiendo retos a nivel profesional y las implicaciones para la formación inicial de maestros en Colombia.

\section{Caracterización de los usos orales de los niños de la Escuela Maternal de la UPN}

Con respecto a estos aprendizajes, la investigación permitió reconocer que el proceso de crecimiento de la referencia llega a ser un interés preponderante para los niños en los primeros años y para ello encuentran usos orales que les posibilitan involucrarse y ser partícipes de situaciones comunicativas como el rasgo deíctico, la onomatopeya, la repetición literal y la pregunta.

Estos usos orales contribuyen sustancialmente a la construcción y ampliación de la referencia, aquella negociación de significados a partir de la cual se consolida con mayor delimitación objetos, animales, situaciones que convergen en el mundo, de allí que se reconozca como el lenguaje y la cognición mantienen una relación estrecha y permanente, tal como lo expone Vygotsky citado por Garton (1994, p. 15) "el lenguaje es un componente necesario en el desarrollo cognitivo. Concretamente, el lenguaje es entendido como un sistema de representación, un sistema que media en el desarrollo cognitivo". Es el 
medio por el cual se establece un saber sobre las cosas, permitiendo realizar asociaciones y discriminaciones de aquello a lo que se vienen acercando.

De esta manera, es de resaltar como los usos orales y los sistemas de apoyo que realiza el maestro permiten que los niños en edades iniciales hagan un reconocimiento de manera paulatina de un Otro quien es parte importante de su construcción como sujeto, vislumbrando con ello, la intersubjetividad como un proceso en el que los niños se involucran de forma progresiva a partir de conversaciones sobre las situaciones de conflicto con sus pares, siendo la mediación de la maestra a través de su palabra, un puente gracias al cual logran acercarse al otro en su necesidad por evidenciar emociones y establecer límites, interiorizando prácticas de cuidado a partir del uso de su oralidad.

\section{Sistemas de apoyo de las maestras de la Escuela Maternal de la UPN}

Es en este marco de la investigación que se identifica el préstamo de conciencia a través de la oralidad de las maestras como primer sistema de apoyo para promover la oralidad de los niños de la Escuela Maternal. Apoyo que se configura a su vez por la pregunta, la reformulación, la expansión semántica y la recapitulación como movimiento dialógico, desde los cuales se evidencia el papel del locutor e interlocutor. Es desde este tipo de interacción que se posiciona el diálogo como medio idóneo por el cual se realizan intercambios verbales y no verbales, favoreciendo el desarrollo de la oralidad.

Las maestras en este sentido, al ser conscientes de su importancia, reestructuran su quehacer pedagógico para, desde allí, enriquecer el proceso formativo de los niños, en el que ponen en juego el préstamo de conciencia a través de su oralidad y contribuyen de manera significativa e intencional en la consolidación de la alteridad. Se da un lugar protagónico a los niños en este proceso y se movilizan para asumir un rol cada vez más activo en el reconocimiento de quienes le acompañan.

De esta manera, es a través del préstamo de conciencia que se favorece la intersubjetividad; la misma se posiciona como un saber al que el maestro debe propender, siendo el lenguaje oral el sistema de referencia por el cual llevar a los más nuevos a posicionarse en el escenario social a través de las búsquedas alrededor de su oralidad. Eso permite movilizarlos a conquistar la palabra en perspectiva de quienes le acompañan, encontrando en ello la oportunidad de construir vínculos sanos y cuidadosos con los otros.

Finalmente, es importante reconocer, gracias a la investigación, que si bien es fundamental llegar a comprender que los contextos familiares son fundamentales en la adquisición del lenguaje de los niños, es pertinente a su vez, contemplar otros escenarios sociales en los que interactúan y se ven motivados a hacer uso del lenguaje. Por esa razón, la Escuela Maternal, como escenario de socialización, se convierte en un contexto crucial en las posibilidades que los niños encuentran para participar de sus propios procesos orales y verse jalonados a elaboraciones más complejas desde el trabajo pedagógico de la oralidad por parte de las maestras participes de esta investigación. 


\section{Implicaciones de los hallazgos de la investigación para la formación inicial de los maestros}

La reflexión se posiciona en la investigación como un dispositivo fundamental en la práctica del maestro, ya que permite visibilizar de manera conceptualizada lo que antes era obvio y complejizar maneras de asumir la enseñanza de la lengua materna o desestructurar perspectivas como las de oralidad, primera infancia y el rol del maestro, seguridades que al ser fisuradas posibilitan continuar aprendiendo. Esto es evidente en las reflexiones y seguimiento a los conversatorios logrando reconocer como cada momento de la ruta metodológica dio la posibilidad para que las maestras hicieran un cambio, consolidaran un aprendizaje diferente al culminar cada paso de la investigación y se convirtieran en la base fundamental para llevar a cabo la acción pedagógica por medio de la cual se impactaría la consolidación de la oralidad en los niños y las niñas de primera infancia de la Escuela Maternal.

Por ello, en esta investigación se resalta el conversatorio como dispositivo que favoreció la cualificación y formación de los maestros en ejercicio dado que no es suficiente con tener claridades teóricas o prácticas; es necesario poder compartir con los pares, aprender desde el contexto mismo a través de lo que se reconoce en el quehacer pedagógico de cada maestra, a partir de sus claridades pedagógicas, didácticas, disciplinares pero también por medio de sus cuestionamientos, dudas, reflexiones, miedos y certezas. En consecuencia, a través de esta investigación, hoy puede afirmarse que, desde una perspectiva interaccionista, cuando se plantea la existencia de un aprendizaje autónomo es porque se han abierto y potenciado en el sistema didáctico de la institución educativa espacios dialógicos para que los maestros aprendan de y con los otros, logrando reflexiones en distintos niveles en el ser y hacer de su rol.
En definitiva, trastocar la mirada frente el trabajo pedagógico de la oralidad debe trascender la enseñanza mecánica de la palabra hablada para convertirse en una oportunidad de participar en diferentes contextos comunicativos y validar a los otros en este marco. La conciencia de ello permitirá otorgar un rol activo a los niños de primera infancia, distando de subestimarlos en sus capacidades. También, ubicar a las maestras como figuras referenciales fundamentales en el andamiaje de los procesos del lenguaje de éstos, contribuyendo a que la conquista de la oralidad sea concebida como una vía potente en la construcción de sociedad y la convivencia sana entre quienes la habitan.

\section{Referencias}

Baena, L.A. (1996). Funciones del lenguaje y enseñanza de la lengua. Revista Lenguaje, 24, 2-10.

Bajtín, M.M. (1998). Estética de la creación verbal. México: Siglo XXI.

Bruner, J. (2001). Acción, pensamiento y lenguaje. Madrid: Alianza.

Camps, A. (2004). Secuencias didácticas para aprender a escribir. Barcelona: Grao.

Cestero, A.M. (1998). Estudios de comunicación no verbal. De la investigación a la práctica en el aula. España: Universidad de Alcalá.

Garton, A. (1994). Interacción social y desarrollo del lenguaje y la cognición. Barcelona: Ediciones Paidós Ibérica, S.A.

Giroux, H. (1987). La formación del profesorado y la ideología del control social. Revista de educación, 284, 53-76. 
Halliday, M. (2013). El lenguaje como semiótica social. La interpretación social del lenguaje y del significado. España: Fondo de Cultura Económica.

Jolibert, J. (1999). Transformar la formación docente. La Didáctica como campo propio, campo de acción y campo de investigación. Chile: Editorial Santillana.

Litwin, E. (1997). Las Configuraciones Didácticas. Una nueva agenda para la Enseñanza Superior. Buenos Aires: Paidós.

Lomas, C. (2006). Usos orales en la escuela. Enseñar lenguaje para aprender a comunicar (se), Volumen I, 79-83

Marcelo, C. (2008). El profesor principiante, inserción a la docencia. Barcelona: Octaedro.

Núñez, M. P. (2003). Didáctica de la comunicación oral: Bases teóricas y orientaciones metodológicas para el desarrollo de la competencia discursiva oral en la educación obligatoria. España: Grupo Editorial Universitario.

Perrenoud, P. (2006). Desarrollar la práctica reflexiva en el oficio de enseñar. Barcelona: Graó.

Reyes, Y. (2010). Ejes de Trabajo Pedagógico: Comunicación Oral. Lineamiento Pedagógico y Curricular para la Educación Inicial en el Distrito, $119-122$.

Silvestri, A y Blanck, G. (1993). Bajtin y Vigostky: la organización semiótica de la conciencia. Barcelona: Anthropos.

Schon, D. (1987). La formación de profesionales reflexivos. Hacia un nuevo diseño de la enseñanza y el aprendizaje en las profesiones. España: Paidós.

Tardif, M. (2004). Los Saberes del docente y su desarrollo profesional. Madrid: Narcea.
Tough, J. (1996). El lenguaje oral en la escuela. Madrid: Editorial Visor.

Tuson, A. (2001). Iguales ante la lengua, desiguales en el uso. Enseñar lenguaje para aprender a comunicar(se). Volumen I, 103-117.

Van Dijk, T. A. (1978). La ciencia del texto. Barcelona: Paidós.

Vigotsky, L. (2000). El desarrollo de los procesos psicológicos superiores. Barcelona: Crítica 